\title{
QUALITY-OF-LIFE IMPROVEMENT COMPARED AFTER HIP AND KNEE REPLACEMENT
}

\author{
F. H. NORMAN-TAYLOR， C. R. PALMER， R. N. VILLAR \\ From Addenbrooke's Hospital NHS Trust and the University of Cambridge, \\ Cambridge, England
}

$\mathbf{T}^{\mathrm{o}}$ otal hip and total knee arthroplasty both provide a considerable improvement in quality of life, but there is no evidence to suggest that one is more successful than the other. We studied 72 patients in a prospective trial before and after total hip or total knee replacement. We recorded scores for disability and distress derived from the Harris hip score and the British Orthopaedic Association knee assessment score, and used them to generate quality of life (QoL) scores using the Rosser Index Matrix immediately before and at one year after surgery.

The patients awaiting knee replacement had significantly lower QoL scores than those awaiting hip replacement $(p=0.011)$. The QoL scores at one year were high and almost identical for both groups $(p=0.46)$. Further analysis showed that gender and weight were not significant predictors of improvement of QoL scores, but age $(p=0.03)$ and whether the hip or knee was replaced $(p=0.006)$ were significant factors.

J Bone Joint Surg [Br] 1996;78-B:74-7.

Received 3 February 1995; Accepted after revision 11 July 1995

Hip replacement is a highly successful operation in terms of improvement in quality of life and cost-effectiveness (Charnley 1972; Wilcock 1978; O’Boyle et al 1992; Laupacis et al 1993; Rorabeck et al 1994). Of all the treatments studied to date Williams (1985) found that hip replacement produced the greatest improvement in quality of life for its cost. Knee replacement has now gained a similar reputation, but has been less extensively studied. Drewett, Minns

F. H. Norman-Taylor, FRCS, Orthopaedic Registrar

R. N. Villar, MS, FRCS, Consultant Orthopaedic Surgeon

Clinic 1, Box 37, Addenbrooke's Hospital NHS Trust, Hills Road, Cambridge CB2 2QQ, UK.

C. R. Palmer, MS, PhD, Medical Statistician

Department of Community Medicine, Institute of Public Health, University of Cambridge, Robinson Way, Cambridge CB2 2SR, UK.

Correspondence should be sent to Mr F. H. Norman-Taylor.

(C)1996 British Editorial Society of Bone and Joint Surgery 0301-620X/96/11119\$2.00 and Sibly (1992) reported the quality of life of 26 patients before and after knee replacement, but were unable to demonstrate an improvement similar to that after hip replacement. Larger series, however, have suggested that hip and knee replacements are comparable in terms of both cost-effectiveness (Liang et al 1986) and reduction of pain and disability scores (Kirwan et al 1994).

Quality of life is recognised as an important outcome measure in orthopaedic replacement surgery. It focuses on health as perceived by the patient, rather than on the status of the prosthesis or other technical concerns which may not be directly related (Gartland 1988). Its numerical value (QoL) can be generated from a qualitative patient-derived classification of health states called the Rosser Index Matrix (Rosser and Watts 1972) (Table I). The classification uses two dimensions, disability and distress, which when combined give 29 possible health states, each with a numerical score for quality of life (Gudex and Kind 1988) (Table II). This index is the one most commonly used in the United Kingdom (Coast 1992).

Data can be collected in three ways: by the use of patient-completed disability/distress questionnaires; by eliciting the views of reference groups, such as clinicians; or by the reprocessing of disease-specific data (Gudex and Kind 1988). The last method is the most useful in the present context since it ensures that the results reflect the

Table I. The Rosser Index (Rosser and Watts 1972)

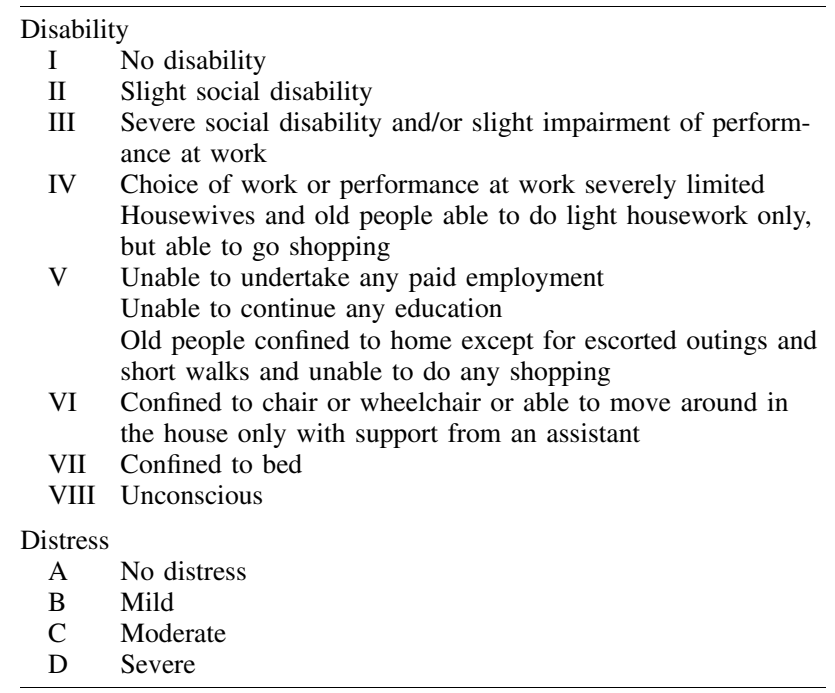


Table II. The Rosser Index Matrix showing a QoL score for each disability/distress combination (Gudex and Kind 1988)

\begin{tabular}{lllll}
\hline \multirow{2}{*}{ Disability } & \multicolumn{4}{l}{ Distress } \\
\cline { 2 - 5 } & A & B & C & D \\
\hline I & 1.000 & 0.995 & 0.990 & 0.967 \\
II & 0.990 & 0.986 & 0.973 & 0.932 \\
III & 0.980 & 0.972 & 0.956 & 0.912 \\
IV & 0.964 & 0.956 & 0.942 & 0.870 \\
V & 0.946 & 0.935 & 0.900 & 0.700 \\
VI & 0.875 & 0.845 & 0.680 & 0.000 \\
VII & 0.677 & 0.564 & 0.000 & -1.486 \\
VIII & -1.028 & & & \\
\hline
\end{tabular}

outcome of the intervention being studied, provided that the data are suitable for reprocessing (Coast 1992).

The QALY (Quality Adjusted Life Year) has an established theoretical role in resource allocation (Williams 1985; Gudex and Williams 1990; Coast 1992), since it scores quantity as well as quality of life, adjusting life expectancy for the quality measured. It is derived from the QoL using assumptions about the life expectancy of the population being studied. Assumptions must also be made about the duration of the benefit achieved and about the quantity of life which a patient may be willing to sacrifice to improve quality (Williams 1985; Coast 1992).

Our aim was to quantify and compare the early impact of hip and knee prosthetic surgery on the quality of life, without making the above assumptions on life expectancy and duration of benefit. We have chosen therefore not to convert the QoL scores into QALYs, although others may wish to do so.

\section{PATIENTS AND METHODS}

A total of 41 patients scheduled for primary Charnley total hip arthroplasty (THA) and 31 for primary Press Fit Condylar (PFC) total knee arthroplasty (TKA) were entered into the study between November 1991 and June 1993. The mean age of the THA patients was 73 years $(\mathrm{SD}=7.2)$ and of the TKA patients 73 years $(\mathrm{SD}=5.1)$. The female-tomale ratios were $1.3: 1$ and $1.7: 1$ respectively. The indication for surgery in all cases was osteoarthritis. All operations were performed either by or under the supervision of the senior author (RNV). All patients were included, regardless of the ease of the procedure or any complications. Follow-up was for one year.

Preoperative pain and function scores were recorded using a modified Harris hip score (Harris 1969) and a modification of the British Orthopaedic Association knee function assessment chart (Aichroth et al 1978). These quantify patient-derived information to produce numerical scores for pain and function. Their qualitative nature allows them to be translated directly into Rosser distress (pain) and disability (function) categories (Table I), which can then be applied to the Rosser Index Matrix (Table II) to derive QoL scores. We excluded questions on deformity because answers to these are not patient-derived.

The Rosser Index Matrix allocates scores from -1.486 to 1.000. A score of 1.000 indicates complete normality, and death is given a score of 0.000 . Negative scores indicate health states thought to be worse than death. The preoperative weight of all patients was recorded as a possible confounding factor.

We obtained postoperative information at one year, using a postal questionnaire, followed by a telephone call if necessary. The data were processed in the same manner as the preoperative scores.

Questionnaires are known to be effective for the collection of reproducible data of this type (Deyo 1991). We did not follow up patients for more than one year as most of the benefits of surgery should have been achieved by then (Pugh 1973). Each patient was given a preoperative and a one-year postoperative QoL score.

Because of the skewed nature of the data, medians are quoted as well as means and non-parametric tests were used to assess the significance of the differences both within and between the hip and knee replacement groups. Within-group comparisons were used to compare preoperative and postoperative scores. Between-group comparisons were used to assess whether improvement differed between one operation and the other. Finally, a multiple-regression analysis on log-transformed preoperative and postoperative differences was used to quantify improvements while allowing adjustments to be made for age, gender and weight, in addition to THA or TKA.

\section{RESULTS}

The response rate at one year was $100 \%$ for both groups, although six patients required reminders. Scores for pain and function derived from the hip and knee scoring systems were reprocessed to provide QoL scores. No patient scored less than 0.700 preoperatively or less than 0.956 at one year.

All patients increased their QoL scores after surgery. Movement across the Index Matrix is illustrated in Tables III to VI. After one year $32 \%$ of patients with a TKA and $39 \%$ of patients with a THA achieved scores of 1.000 . The median preoperative score was 0.942 for THA (range 0.700 to 0.973 ; mean 0.928 ) and 0.912 for TKA (range 0.700 to 0.973 ; mean 0.889). The median postoperative score was 0.990 for THA (range 0.956 to 1.000 ; mean 0.991 ) and 0.990 for TKA (range 0.956 to 1.000 ; mean 0.990 ). The median increase in score for THA was 0.048 (0.007 to $0.300)$ with a mean of 0.063 (95\% confidence interval (CI) 0.046 to 0.080$)$. The median increase in score for TKA was 0.083 (0.013 to 0.300$)$ with a mean of 0.101 (95\% CI 0.071 to 0.131 ).

The differences in preoperative QoL scores between 
Table III. Distribution of 31 patients in the Rosser Index Matrix before TKA

\begin{tabular}{lllll}
\hline & \multicolumn{4}{l}{ Distress } \\
\cline { 2 - 5 } Disability & A & B & C & D \\
\hline I & - & - & - & - \\
II & - & - & 2 & 2 \\
III & - & - & 6 & 10 \\
IV & - & - & - & 7 \\
V & - & - & - & 4 \\
VI & - & - & - & - \\
\hline
\end{tabular}

Table IV. Distribution of 31 patients in the Rosser Index Matrix one year after TKA

\begin{tabular}{lllll}
\hline & \multicolumn{4}{l}{ Distress } \\
\cline { 2 - 5 } Disability & A & B & C & D \\
\hline I & 10 & 4 & - & - \\
II & 4 & 9 & 1 & - \\
III & - & 2 & 1 & - \\
IV & - & - & - & - \\
V & - & - & - & - \\
VI & - & - & - & - \\
\hline
\end{tabular}

Table V. Distribution of 41 patients in the Rosser Index Matrix before THA

\begin{tabular}{lllll}
\hline & \multicolumn{4}{l}{ Distress } \\
\cline { 2 - 5 } Disability & A & B & C & D \\
\hline I & - & - & - & - \\
II & - & - & 15 & 2 \\
III & - & - & 3 & 8 \\
IV & - & - & 4 & 8 \\
V & - & - & - & 1 \\
VI & - & - & - & - \\
\hline
\end{tabular}

Table VI. Distribution of 41 patients in the Rosser Index Matrix one year after THA

\begin{tabular}{lllll}
\hline & \multicolumn{4}{l}{ Distress } \\
\cline { 2 - 5 } Disability & A & B & C & D \\
\hline I & 16 & 3 & 3 & - \\
II & 8 & 5 & 2 & - \\
III & 2 & 1 & - & - \\
IV & - & 1 & - & - \\
V & - & - & - & - \\
VI & - & - & - & - \\
\hline
\end{tabular}

THA and TKA patients were significant using a Wilcoxon rank-sum test $(p=0.011)$ with a mean difference in improvement between groups of 0.038 (95\% CI 0.006 to 0.069). The QoL scores, however, were increased more by TKA than by THA $(\mathrm{p}=0.013)$ so that the postoperative scores were effectively identical $(p=0.47)$.
Multiple-regression analysis showed that neither weight $(p=0.36)$ nor gender $(p=0.39)$ were significant in affecting improvement in QoL scores but that age $(\mathrm{p}=0.028)$ and site of arthroplasty $(\mathrm{p}=0.006)$ were. The best predictive model was found to be:

Improvement $=\exp (0.03 \mathrm{X}$ age -0.5 (if hip arthroplasty $)-4.77$

(where 'exp' denotes exponentiation to base e). For example, for a patient aged 70 years, the improvement in QoL score would be expected to be 0.069 for a knee replacement and 0.042 for a hip replacement.

\section{DISCUSSION}

The translation of existing scoring systems into Rosser health state categories, although widely performed, has been criticised as a potential source of error and subjectivity (Carr-Hill and Morris 1991; Coast 1992). Certainly, the comparison of historical QoL scores derived independently by secondary data analysis is probably not valid, because of the subjective judgements involved. Table II shows that allocation to the wrong category in the matrix can considerably alter the QoL score.

Both the hip and knee scores used in this study, however, lend themselves well to translation, allowing the patient to answer questions relevant to his or her joint disease and operation, while using the same variables of pain (distress) and function (disability) as each other and the Rosser categories.

It is therefore not clear why patients awaiting knee replacement have a poorer quality of life than those awaiting hip replacement, although disability (function) contributes most of this difference. Differences in pain and disability were not reported by Kirwan et al (1994). When selecting patients for surgery there may be a bias towards patients requiring hip replacement particularly in view of recent pressures to give hip replacement a priority on waiting lists. This may not be unique to the reporting orthopaedic unit, and has implications for resource allocation. The patients reported by Drewett et al (1992) had a median preoperative QoL score of 0.912 (0.700 to 0.986) and a median QoL score at least two months after surgery of 0.980 (0.900 to 1.000). These results are very similar to those which we report.

It had been predicted that knee replacement would prove as valuable as hip replacement in improving quality of life (Newman 1992) and our results show this to be the case. We showed a return to normality $(\mathrm{QoL}=1.000)$ in $39 \%$ of patients after THA and in 32\% of patients after TKA. All our patients increased their QoL scores, but there was a greater increase after TKA which could be entirely explained by the lower baseline of preoperative scores. It may be more cost-effective to treat patients with lower preoperative health scores (Liang et al 1986) since the postoperative scores were effectively identical. It is evident 
that both hip and knee replacement arthroplasty are both highly and equally effective at improving quality of life.

No benefits in any form have been received or will be received from a commercial party related directly or indirectly to the subject of this article.

\section{REFERENCES}

Aichroth P, Freeman MAR, Smillie IS, Souter WA. A knee function assessment chart. J Bone Joint Surg [Br] 1978;60-B:308-9.

Carr-Hill RA, Morris J. Current practice in obtaining the 'Q' in QALYs: a cautionary note. Br Med J 1991;303:699-701.

Charnley J. The long-term results of low-friction arthroplasty of the hip performed as a primary intervention. J Bone Joint Surg [Br] 1972; 54-B:61-76.

Coast J. Reprocessing data to form QALYs. Br Med J 1992;305:87-90.

Deyo RA. The quality of life, research and care. Ann Intern Med 1991;114:695-7.

Drewett RF, Minns RJ, Sibly TF. Measuring outcome of total knee replacement using quality of life indices. Ann $R$ Coll Surg Engl 1992;74:286-9.

Gartland JJ. Orthopaedic clinical research: deficiencies in experimental design and determinations of outcome. J Bone Joint Surg [Am] 1988; 70-A:1357-64.

Gudex C, Kind P. The QALY Toolkit. Discussion paper 38. University of York: Centre for Health Economics, 1988.

Gudex C, Williams A. Prioritising waiting lists. Health Trends 1990; 22:103-8.
Harris WH. Traumatic arthritis of the hip after dislocation and acetabular fractures: treatment by mold arthroplasty. J Bone Joint Surg [Am] 1969;51-A:737-55.

Kirwan JR, Currey HL, Freeman MA, Snow S, Young PJ. Overall long-term impact of total hip and knee joint replacement surgery on patients with osteoarthritis and rheumatoid arthritis. $\mathrm{Br}$ J Rheumatol 1994:33:357-60.

Laupacis A, Bourne R, Rorabeck C, et al. The effect of elective total hip replacement on health-related quality of life. J Bone Joint Surg [Am] 1993;75-A:1619-26.

Liang MH, Cullen KE, Larson MG, et al. Cost-effectiveness of total joint arthroplasty in osteoarthritis. Arthritis Rheum 1986;29:937-43.

Newman JH. Invited comment. Ann R Coll Surg Engl 1992;7:289-90.

O'Boyle CA, McGee H, Hickey A, O'Malley K, Joyce CR. Individual quality of life in patients undergoing hip replacement. Lancet 1992;339:1088-91.

Pugh LGCE. The oxygen intake and energy cost of walking before and after unilateral hip replacement, with some observations on the use of crutches. J Bone Joint Surg [Br] 1973;55-B:742-5.

Rorabeck CH, Bourne RB, Laupacis A, et al. A double-blind study of 250 cases comparing cemented with cementless total hip arthroplasty: cost-effectiveness and its impact on health-related quality of life. Clin Orthop 1994;298:156-64.

Rosser RM, Watts VC. The measurement of hospital output. Int J Epidemiol 1972;1:361-8.

Wilcock GK. Benefits of total hip replacement to older patients and the community. Br Med J 1978;2:37-9.

Williams A. Economics of coronary artery bypass grafting. $\mathrm{Br} \mathrm{Med} \mathrm{J}$ $1985 ; 291: 326-9$ 